\title{
A REMARK ABOUT A GALERKIN METHOD
}

\author{
GERHARD STRÖHMER \\ Department of Mathematics, University of Iowa \\ Iowa City, Iowa 52242, U.S.A.
}

\begin{abstract}
It is shown that the approximating equations whose existence is required in the author's previous work on partially regular weak solutions can be constructed without any additional assumption about the equation itself. This leads to a variation of a Galerkin method.

In the paper [1] the existence of partially regular weak solutions of an abstract parabolic equation of the form

$$
U_{t}+A U=F(U, t)
$$

is proved under the assumption that there exists a family of approximating equations

$$
U_{t}+A U=F_{k}(U, t)
$$

for $k=1,2, \ldots$ which are strongly solvable. Here we show that such an approximating sequence always exists, if we just assume what is directly implied in the assumptions for the equation $(E Q)$. For further literature and some applications see both [1] and [2]. We adhere to the notation used in [1], with the exception of denoting the real numbers by $R$ and the non-negative real numbers by $R^{+}$. Now we make the following assumptions.

Let $A$ denote a self-adjoint positive operator on a Hilbert space $H$ with domain of definition $D(A)$ which has a compact inverse $A^{-1}$. Let $T \leq+\infty$ and $I=[0, T]$ for $T<+\infty$, while $I=[0,+\infty)$ otherwise. Assume also that with the fixed number $\mu \in(0,1)$
\end{abstract} the nonlinearity $F: D(A) \times I \rightarrow H$ has the following properties.

1. There are numbers $\eta<1$ and $C_{1}<\infty$ such that for all $U \in D(A), t \in I$ we have with $a^{+}=\max (a, 0)$ and $a^{-}=\max (-a, 0)$

$$
\{(F(U, t), U)\}^{+} \leq \eta\left[\{(F(U, t), U)\}^{-}+\left\|A^{1 / 2} U\right\|^{2}\right]+C_{1}\left(\|U\|^{2}+1\right) .
$$

2. There is a continuous increasing function $C_{2}: R^{+} \rightarrow R^{+}$such that for $U, U^{\prime} \in$ $D(A), t, t^{\prime} \in I$ we have

$$
\left\|F(U, t)-F\left(U^{\prime}, t^{\prime}\right)\right\| \leq C_{2}\left(\left\|A^{\mu} U\right\|+\left\|A^{\mu} U^{\prime}\right\|\right)\left(\left\|A^{\mu}\left(U-U^{\prime}\right)\right\|+\left|t-t^{\prime}\right|\right) \quad \text { and }
$$

1991 Mathematics Subject Classification: 34G20, 35Q30.

The paper is in final form and no version of it will be published elsewhere. 


$$
\|F(U, t)\| \leq C_{2}\left(\left\|A^{\mu / 2} U\right\|\right)\left(\left\|A^{\mu} U\right\|+1\right) .
$$

3. There is a continuous increasing function $C_{3}: R^{+} \rightarrow R^{+}$such that for all $U, V \in$ $D(A), t \in I$ we have

$$
|(F(U, t), V)| \leq C_{3}(\|U\|)\|A V\|\left(\left\|A^{1 / 2} U\right\|^{2 \mu}+\left((F(U, t), U)^{-}\right)^{\mu}+1\right) .
$$

The notation for function spaces in this paper does not differ much from general practice, and coincides completely with that used in [1]. We call a function $U:\left(t, t^{\prime}\right) \rightarrow H$ a strong solution of the equation $(E Q)$ on the interval $\left(t, t^{\prime}\right)$ if $U \in C^{1}\left(\left(t, t^{\prime}\right), H\right) \cap$ $C^{0}\left(\left(t, t^{\prime}\right), D(A)\right)$ and fulfills the equation there. Then we get the following slightly enhanced version of the main theorem of [1].

Theorem. For every $\mathcal{U}_{0} \in H$ there is a function $U: I \rightarrow H$ such that $U \in$ $L^{\infty}((0, \widehat{T}), H) \cap L^{2}\left((0, \widehat{T}), D\left(A^{1 / 2}\right)\right)$ for all $\widehat{T} \leq T, \widehat{T} \in R$, which also has the following properties.

$(P 1)$ We have $A^{-1} U(t) \in C^{0}([0, \widehat{T}], H)$ and $U(0)=\mathcal{U}_{0}$. There is a relatively open set $\mathcal{D} \subset[0, \widehat{T}]$ such that $m([0, \widehat{T}] \backslash \mathcal{D})=0$, and $U \in C^{1}(\mathcal{D}, H) \cap C^{0}(\mathcal{D}, D(A))$, and

$$
U_{t}+A U=F(U, t)
$$

for $t \in \mathcal{D}$. We have $0 \in \mathcal{D}$ if $\mathcal{U}_{0} \in D(A)$, and $\left\|A^{\mu / 2} U(t)\right\| \rightarrow \infty$ as $t \uparrow \partial \mathcal{D} \backslash\{0, \widehat{T}\}$.

$(P 2)$ In addition $U$ is a weak solution of our problem, i.e. for $V \in C^{1}([0, \widehat{T}], H) \cap$ $C^{0}([0, \widehat{T}], D(A))$ we have

$$
\int_{\mathcal{D} \cap\left(t, t^{\prime}\right)}-(U, A V)+(F(U, t), V)+\left(U, V_{t}\right) d \tau=\left(U\left(t^{\prime}\right), V\left(t^{\prime}\right)\right)-(U(t), V(t))
$$

for $0 \leq t \leq t^{\prime} \leq \widehat{T}$.

(P3) There is an $\varepsilon_{0}>0$ such that if $\|U(t)\| \leq \varepsilon \leq \varepsilon_{0}$ in $\left[T_{1}, T_{2}\right] \subset[0, \widehat{T}]$, then $\left[T_{1}+\varepsilon^{2}, T_{2}\right] \subset \mathcal{D}$. Also for all $t \in[0, \widehat{T}]$ we have

$$
\begin{gathered}
\|U(t)\|^{2} \leq \exp (2 \alpha t)\|U(0)\|^{2}+C_{1}(\exp (2 \alpha t)-1) / \alpha \quad \text { for } \alpha \neq 0 \quad \text { and } \\
\|U(t)\|^{2} \leq\|U(0)\|^{2}+2 C_{1} t \quad \text { for } \alpha=0,
\end{gathered}
$$

where $\alpha=C_{1}-(1-\eta) \lambda_{1}$, with $\lambda_{1}$ being the lowest eigenvalue of $A$.

Therefore, if $C_{1}$ is sufficiently small, this solution will become strong after a time determined by $C_{1}$ and $\left\|\mathcal{U}_{0}\right\|$, and if both $C_{1}$ and $\left\|A \mathcal{U}_{0}\right\|$ are small enough, it is strong on the whole interval. The former is also true if $C_{1}$ only becomes small after a time. Also if for a $t_{0} \in \mathcal{D}$ there is a strong solution $V(t)$ of the initial-value problem on $\left[t_{0}, \widehat{T}\right]$, and $V\left(t_{0}\right)=U\left(t_{0}\right)$, then $U(t)=V(t)$ for $t \geq t_{0}$.

Now we can prove our theorem. First it is clear by checking the proof of Theorem 1.1 in [1] that we do not really need to assume $\mathcal{U}_{0} \in D(A)$. Instead we can also approximate $\mathcal{U}_{0}$ from this space and then proceed as before, just losing the fact that $0 \in \mathcal{D}$. Now we have to find a series of approximating equations and verify the assumptions of [1]. We use $F_{k}(U, t)=P_{k}\left(F\left(P_{k} U, t\right)\right)$ where $P_{k}$ is the orthogonal projection of $H$ to the space generated by the first $k$ eigenfunctions of $A$. Then we have of course $\left\|P_{k} U\right\| \leq\|U\|$ for 
all $U \in H$ and $\left\|A^{s} P_{k} U\right\| \leq\left\|A^{s} U\right\|$ for all $U \in D\left(A^{s}\right), s>0$. This makes it clear that hypothesis $(H 2)$ in $[1]$ is fulfilled. For $(H 1)$ note that

$$
\begin{aligned}
\left\{\left(P_{k} F\left(P_{k}(U), t\right), U\right)\right\}^{+} & =\left\{\left(F\left(P_{k}(U), t\right), P_{k} U\right)\right\}^{+} \\
& \leq \eta\left[\left\{\left(F\left(P_{k}(U), t\right), P_{k}(U)\right)\right\}^{-}+\left\|A^{1 / 2} P_{k} U\right\|^{2}\right]+C_{1}\left(\left\|P_{k} U\right\|^{2}+1\right) \\
& \leq \eta\left[\left\{\left(P_{k} F\left(P_{k} U, t\right), U\right)\right\}^{-}+\left\|A^{1 / 2} U\right\|^{2}\right]+C_{1}\left(\|U\|^{2}+1\right) \\
& =\eta\left[\left\{\left(F_{k}(U, t), U\right)\right\}^{-}+\left\|A^{1 / 2} U\right\|^{2}\right]+C_{1}\left(\|U\|^{2}+1\right) .
\end{aligned}
$$

For $(H 3)$ consider

$$
\begin{aligned}
\left|\left(P_{k} F\left(P_{k} U, t\right), V\right)\right| & =\left|\left(F\left(P_{k} U, t\right), P_{k} V\right)\right| \\
& \leq C_{3}\left(\left\|P_{k} U\right\|\right)\left\|A P_{k} V\right\|\left(\left\|A^{1 / 2} P_{k} U\right\|^{2 \mu}+\left(\left(F\left(P_{k} U, t\right), P_{k} U\right)^{-}\right)^{\mu}+1\right) \\
& \leq C_{3}(\|U\|)\|A V\|\left(\left\|A^{1 / 2} U\right\|^{2 \mu}+\left(\left(F_{k}(U, t), U\right)^{-}\right)^{\mu}+1\right) .
\end{aligned}
$$

Now the inequality in $(H 4)$ is even fulfilled in the very strong form

$$
\left\|F_{k}(U, t)\right\| \leq C_{k}^{*}(\|U\|)
$$

which is very easy to ascertain as $\left\|A^{\mu} P_{k} U\right\| \leq C_{k}\|U\|$. The convergence $F_{k}(U, t) \rightarrow$ $F(U, t)$ for $U \in D(A)$ is obvious. So we have now verified all conditions in [1], and therefore proved our theorem. To see the relationship with a Galerkin method apply $P_{k}$ to the equation

$$
U_{k t}+A U_{k}=P_{k} F\left(P_{k} U_{k}, t\right) .
$$

This gives us

$$
\left(P_{k} U_{k}\right)_{t}+A P_{k} U_{k}=P_{k} F\left(P_{k} U_{k}, t\right)
$$

so the evolution of $P_{k} U_{k}$ is exclusively determined by $P_{k} U_{k}$ itself, and it is a Galerkinapproximation for our solution using the eigenfunctions of $A$. If $P_{k}^{*}$ is the projection to the perpendicular space, we get $\left(P_{k}^{*} U_{k}\right)_{t}+A\left(P_{k}^{*} U_{k}\right)=0$, so if our initial value is chosen as belonging to the linear hull of the first $k$ eigenvalues, $U_{k}$ itself even is a Galerkin approximation of our solution.

\section{References}

[1] G. Ströhmer, An existence result for partially regular weak solutions of certain abstract evolution equations, with an application to magneto-hydrodynamics, Math. Z. 213 (1993), 373-385.

[2] - Partially regular weak solutions for a system of equations modeling liquid crystal motion, Analysis 14 (1994), 381-391. 